\title{
Modeling Release Behavior in Shocked Tantalum
}

\author{
Daniel J. Steinberg
}

This paper was prepared for submittal to the 1995 APS Topical Conference on Shock Compression of Condensed Matter Seattle, WA

August 13-18, 1995

August 1995

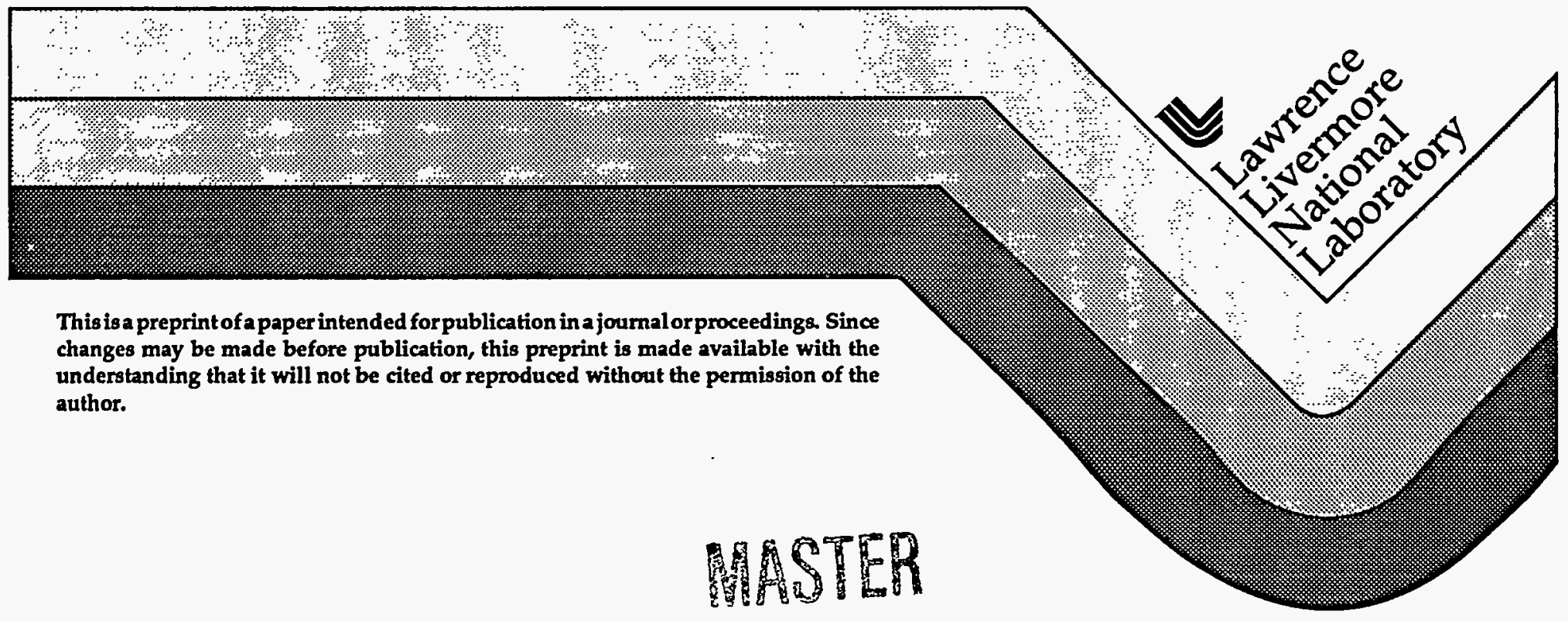

DISTAIRUTION OF THIS DOCUMENT IS UNLIMITED BS 


\section{DISCLAIMER}

This document was prepared as an account of work sponsored by an agency of the United States Government. Neither the United States Government nor the University of California nor any of their employees, makes any warranty, express or implied, or assumes any legal liability or responsibility for the accuracy, completeness, or usefulness of any information, apparatus, product, or process disclosed, or represents that its use would not infringe privately owned rights. Reference herein to any specific commercial product, process, or service by trade name, trademark, manufacturer, or otherwise, does not necessarily constitute or imply its endorsement, recommendation, or favoring by the United States Government or the University of California. The views and opinions of authors expressed herein do not necessarily state or reflect those of the United States Government or the University of California, and shall not be used for advertising or product endorsement purposes. 


\section{DISCLAIMER}

Portions of this document may be illegible in electronic image products. Images are produced from the best available original document. 


\title{
MODELING RELEASE BEHAVIOR IN SHOCKED TANTALUM
}

\author{
Daniel J. Steinberg \\ Lawrence Livermore National Laboratory, PO Box 808 (L35), Livermore, CA 94551, USA
}

\begin{abstract}
Using Johnson's anelastic release model for metals $(3,4)$, hydrocode simulation of four Ta plate-impact experiments were performed. These calculations agree well with the data as long as the complete nonlinear version of the model is used and the yield surface is itself made asymmetric, i.e., different on loading and unloading. From the parameters in the model it is possible to determine the drag coefficient, mobile dislocation density, and characteristic length of a dislocation, and to obtain reasonable values.
\end{abstract}

\section{INTRODUCTION}

It has been demonstrated that the rate-dependence of the yield strength plays a major role in simulating shock smearing or the gradual rise in a shock-loading profile (1). It has been further shown that rate effects can play a dominant role in other, more complex, deformation experiments such as the 3-D formation of an explosively formed projectile (2). Therefore, it seems reasonable to assume that modeling the gradual release of these shocks may be equally important.

In a series of papers Johnson set forth his idea of using a backstress model to describe the quasi elastic or anelastic release behavior of shock-loaded metals $(3,4)$. In some quarters, this behavior is called either the Bauschinger or dynamic Bauschinger effect. In any case, all mean here that the material strength on unloading may not be the same as on loading. This report will describe what I have done to extend Johnson's work. While effort has been placed on a number of materials, I will concentrate here on tantalum.

\section{BASIC EQUATIONS AND ASSUMPTIONS}

Johnson's equations for straight dislocation pileups can be summarized as

$$
\dot{s}=2 G\left[\dot{\varepsilon}-C_{1}(s-\beta)\right] \text {, }
$$

and

$$
\dot{\beta}=\frac{C_{2} \beta^{2}(s-\beta)}{1-\left[1+\left(C_{3} \beta\right)^{2}\right]^{-1 / 2}}
$$

Here. $s$ is the stress deviator, $G$ the shear modulus, $\varepsilon$ the equivalent plastic strain, and $\beta$ the backstress. Dots over these symbols connote time derivatives. The constants $C_{1-3}$ are defined by $C_{1}=n b^{2} / B, C_{2}=$ $b / a B$, and $C_{3}=L / 2 a$, where $a=G b / 2 \pi(1-v)$. The parameter $B$ is the drag coefficient, $n$ is the number of mobile dislocations per unit area, $b$ is the Burgers' vector, $L$ is a characteristic length for this type of dislocation, and $v$ is Poisson's ratio.

Johnson linearized eqn. (2) by assuming $C_{3} \beta \ll 1$, producing

$$
\dot{\beta}=C_{4}(s-\beta)=\left(8 \mathrm{ab} / L^{2} \mathrm{~B}\right)(\mathrm{s}-\beta) .
$$

Then using eqns. (1) and (3), his best fits to shockwave profiles had $B$ about two orders of magnitude too large when compared with theoretical calculations (3). Johnson's data base was limited. When I tried fitting a greater number of experiments, covering a wider range of values for the thermomechanical variables, I also found similar results for B. But, in addition, I was unable to adequately fit any two experiments. As a temporary solution, I locked $\beta$ to $s$ until the hydrostat was crossed. This is similar to the default Cochran-Guinan model (5). Now, B was about the right order of magnitude, and I could fit up to 8 experiments for a given material; typically 3 experiments. Good results were obtained for $\mathrm{Al}, \mathrm{Cu}$, SS 304, Ta, Mo, W, and even Be.

However, there are some definite problems with this approach. In particular, the linearity of eqn. (3) depends on $C_{3} \beta$ \& 1 . Johnson noted that $C_{3} \beta$ could reach 17 in some cases (4), yet he felt that his results were so good that he continued to use eqn. (3) in place of eqn. (2). I also found that $C_{3} \beta$ reached values of

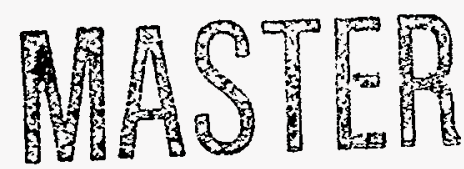


10-20. The obvious solution to this problem is to use eqn. (2) in place of eqn. (3) and to no longer require $\beta$ to be locked to $\mathrm{s}$.

There are many kinds of dislocation configurations which can give rise to backstress and Johnson examined only two simplified cases, that of pinned loops and straight pileups. In the linearized form, these differ only by the factor $2 \pi(1-v) \approx 4$. However, in their non-linear form, only the pileup model mathematically allows $C_{3} \beta$ to exceed unity. Consequently, I have assumed that Johnson's pileup model, in some average sense, as shown by eqn. (2), adequately represents any type or combination of types of dislocations.

\section{COMPARISON WTTH 1-D SHOCK-WAVE DATA}

The data consist of four 1-D plate-impact experiments, two single shock and two double shock, covering a range of pressure from 3.5 to $12 \mathrm{GPa}(6)$. Figures 1-3 compare these experimental wave profiles with calculations. The data in Fig. 1 and the doubleshock data in Fig. 3 were each reduced by $2.2 \%$. However, this is within the absolute accuracy of the VISAR. The parameters for the Grüneisen equationsof state and the constitutive models are the same as those in ref. (7) with the following exceptions for Ta.

The work-hardening parameters have been changed from 10 and 0.1 to 22 and 0.283 based on the recent quasi-isothermal data of Lopatin et al. (8). In addition, the coefficient $\mathrm{B} / \mathrm{nb}^{2}$ has been reduced by a factor of 5 to $2.4 \mathrm{GPa} \bullet \mu \mathrm{s}(0.024 \mathrm{Mb} \bullet \mu \mathrm{s})$. This value is based on a fit to the upper part of the single-shockloading profile in Fig. 3, but is still compatible with all the strain-rate data in ref. (1).

It is clear from Figs. 1 and 2 that the calculations without a Bauschinger effect are inadequate. The sharp drop followed by a plateau bears little resemblance to the gradual falloff seen in the data. With eqns. (1) and (2) and the usual symmetric yield surface, the calculations are now much closer to the experiments. However, they still fall below the data.

Indeed, the agreement in the purely plastic release region is now not as satisfactory as without any Bauschinger effect. This new problem was addressed by making the yield surface itself asymmetric. This was done making the thermally-activated part of the Steinberg-Guinan-Lund (SGL) rate-dependent model (1) vanish when the deviator crossed the hydrostat on release. For the rest of the problem, the yield surface remained at this smaller value.

\section{DISCUSSIÖN}

Evidence for an asymmetric yield surface already exists as shown by the uniaxial tension-compression data of Lassila and LeBlanc (Fig. 4) (9). At a temperature of $4 \mathrm{~K}$, which corresponds to a reasonably high strain-rate, there is a clear decrease in the yield strength when the Ta sample is unloaded. In addition, Frank Nabarro has suggested that while it takes significant energy to move pinned loops during loading, upon release, line tension allows these loops to collapse with much less energy required.

Figure 5 shows the corresponding deviatoric-stress total-strain behavior in the middle of the target for the double-shock experiment in Fig. 2. Curve $A$ is for the calculation with no Bauschinger effect. It shows the classic linear relationship with a slope equal to that of the elastic loading curve. In the symmetric yield case, $B$, the curve begins immediately to deviate from linearity and after crossing the hydrostat, it begins to level out. Thus, the effective shear modulus, $0.5 \mathrm{ds} / \mathrm{d} \varepsilon$, is approaching zero and, therefore, should be approaching the opposite yield surface. However, to actually reach the opposite yield surface, this stressstrain curve must drop rapidly. This puts in the additional structure, not seen in the data, which is reflected in the calculation in Fig. 1. By using an asymmetric yield surface, we can get around this contradictory situation as shown by curve $C$. Now the effective shear modulus goes smoothly to zero and the opposite yield surface simultaneously.

All rate dependence is not turned off on release; the drag term in the SGL model is still operative. If it too were turned off, the plastic release would no longer be smooth; rather one would see a stair-step like release. This again illustrates what has been observed before, namely, that both rate-dependence and the Bauschinger effect are important in smoothing out the calculated release. The effect of the drag term can also be seen in Fig. 5. The flat part of the stress-strain curve is not truly flat, but rather has some structure.

Future work will aim at constructing a more sophisticated asymmetric yield surface, one that is independent of whether the initial loading is tension or compression as well as one that is applicable in multidimensional hydrocodes.

In addition, adding twinning to the yield relation is proposed. It takes energy to form the twins and 
additional energy to move them through the lattice. However, on release, no energy of formation is required; only what is needed to move them back. This is a more physically reasonable approach than changing the Peierls' stress upon release.

Equations. (1) and (2) have three coefficients which allow us to determine $B, n$, and $L$. For these four experiments, $\mathrm{C}_{1}=0.08$ (GPa $\left.\mu \mathrm{s}\right)^{-1}, \mathrm{C}_{2}=500 \mathrm{GPa}^{-2}$. $\mu s^{-1}$, and $\mathrm{C}_{3}=7 \mathrm{GPa}^{-1}$. This implies that $\mathrm{B}=0.1 \mathrm{~Pa} \cdot \mathrm{s}$, $\mathrm{L}=235 \mathrm{~b}$, and $\mathrm{n}=10^{14} \mathrm{~m}^{-2}$. These are reasonable values for $L / b$ and $n$, but $B$ is again about two orders of magnitude too large. If the expressions for $C_{2}$ and $\mathrm{C}_{3}$ are multiplied by the factor $2 \pi(1-v)$, they equal their counterparts for pinned loops. If these expressions are used instead of those for the straight pileups, the results are better. Now $L / b=10^{3}$, $\mathrm{n}=3 \times 10^{13} \mathrm{~m}^{-2}$, and $\mathrm{B}=3 \times 10^{-2} \mathrm{~Pa} \cdot \mathrm{s}$. While the results here are not as tidy as one might wish, they again demonstrates that it may be possible to use macroscopic experiments to determine microscopic properties.

The value of $B / n$ here is 5 times greater than the same ratio used in the SGL model. The value of $B$ is roughly constant, increasing, due to temperature, by at most about $15 \%$ (10). Nabarro believes that it is certainly possible that $\boldsymbol{n}$ could differ by a factor of 5 depending on whether the system is loading or unloading. In any case, determining $n$ in two different ways to within a factor of 5 is, in itself, a useful result, for, most assuredly, $n$ is a complex function of the thermomechanical variables, and not a constant.

\section{CONCLUSIONS}

Johnson's anelastic release model, in its non-linear form, does a good job of reproducing the dynamic Bauschinger effect in shocked Ta. Using the three constants in this model, it is possible to calculate the drag coefficient, mobile dislocation density, and characteristic length of a dislocation, and to obtain reasonable values. To reproduce the entire wave profiles, it appears that an asymmetric yield surface is also required. To accomplish this, the thermallyactivated part of the SGL yield model vanishes on the opposite side of the hydrostat.

\section{REFERENCES}

1. Steinberg, D. J., and Lund, C. M., J. Appl. Phys. 65, 1528-1533 (1989).
2. Baum, D., Honodel, C., and Schneider, D., Effect of Material Strength Models in the Formation Dynamics of a Three-Dimensional Explosively Formed Penetrator: Comparison of Calculations with Experiment, Lawrence Livermore Nat. Lab. Rpt. UCRL-100429, 1989.

3. Johnson, J. N., Lomdahl, P., and Willis, J., Acıa Met. 39, 3015-3026 (1991).

4. Johnson, J. N., Hixson, R., Gray, G. T., and Morris, C., J. Appl. Phys. 72, 429-441 (1992).

5. Steinberg, D. J., Cochran, S., and Guinan, M., J. Appl. Phys. 51, 1498-1504 (1980).

6. Furnish, M. D., Chhabildas, L. C., and Steinberg, D. J., "Dynamical behavior of tantalum," in High Pressure Science and Technology - 1993, 1994, pp. 1099-1102.

7. Steinberg, D. J., Equation of State and Strength Properties of Selected Materials, Lawrence Livermore Nat. Lab. Rpt. UCRL-MA-106439, 1991.

8. Lopatin, C., Wittman, C., Swenson, J., and Perron, P., "Dependency on strain rate path of mechanical properties of tantalum in compression," in High Strain Rate Behavior of Refractory metals and Alloys, 1992, pp. 241247.

9. Lassila, D., and LeBlanc, M., Lawrence Livermore Nat. Lab., unpublished data, (1995).

10. Kocks, U., Argon, A., and Ashby, F., Thermodynamics and Kinetics of Slip. Oxford: Pergamon Press, 1975, p.74.

\section{Figure Captions}

FIGURE 1. Comparison of data with calculation for a single shock to $8 \mathrm{GPa}$. The long dash is for no Bauschinger and the usual symmetric yield surface; the medium dash adds the Bauschinger effect; the solid line adds the asymmetric yield surface. The data are represented by the short dash line.

FIGURE 2. Comparison of data with calculation for a double shock to $5 \mathrm{GPa}$ peak stress. The long dash is for no Bauschinger and the usual symmetric yield surface; the medium dash adds the Bauschinger effect; the solid line adds the asymmetric yield surface. The data are represented by the short dash line.

FIGURE 3. Comparison of data (dashed line) with calculation (solid line) using the Bauschinger model and the asymmetric yield surface for two experiments shocked to about $12 \mathrm{GPa}$.

FIGURE 4. Tension-compression data at 4,77, and $300 \mathrm{~K}$.

FIGURE 5. Plots of deviatoric stress vs total strain in the middle of the Ta target. See text for meanings of labels A-C.

\section{ACKNOWLEDGMENTS}

This work was performed under the auspices of the U.S. Department of Energy by Lawrence Livermore National Laboratory under contract No. W-7405-Eng-48. 

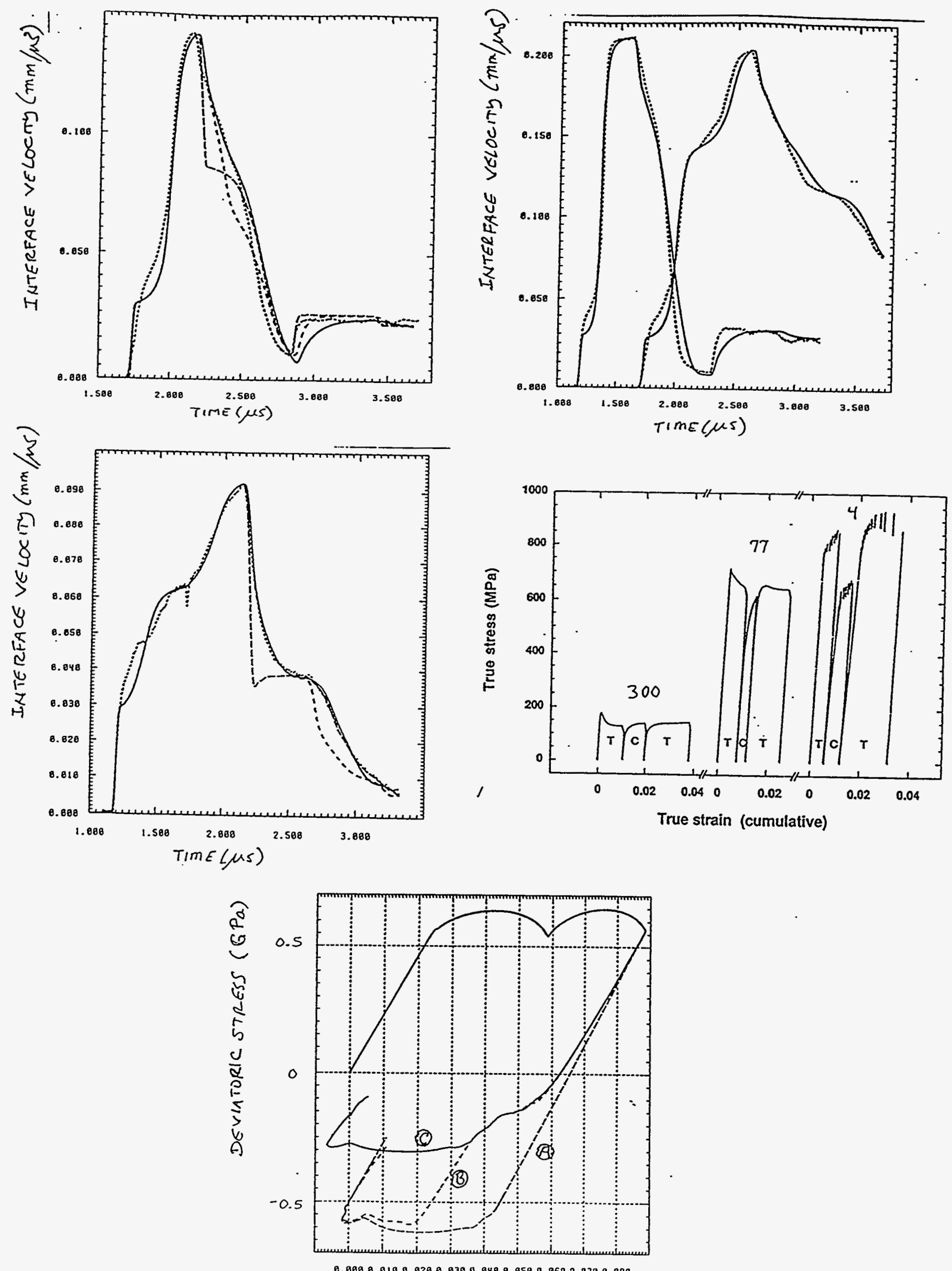